\title{
Spatial learning in pigs: effects of environmental enrichment and individual characteristics on behaviour and performance
}

\author{
Jarno Jansen · J. Elizabeth Bolhuis • \\ Willem G. P. Schouten · Berry M. Spruijt • \\ Victor M. Wiegant
}

Received: 20 April 2007 / Revised: 20 August 2008 / Accepted: 21 August 2008 / Published online: 16 September 2008

(C) The Author(s) 2008. This article is published with open access at Springerlink.com

\begin{abstract}
This study investigated the effects of both environmental enrichment and individual behavioural characteristics on spatial cognitive capabilities of pigs, using a novel latent spatial learning paradigm based on Tolman's detour experiments (1948). Pigs were housed either in 'barren' pens or in pens enriched with straw bedding from birth. Pigs were restrained in a Backtest at 10 and 17 days
\end{abstract}

J. Jansen · J. E. Bolhuis · W. G. P. Schouten · B. M. Spruijt Ethology Group, Wageningen Institute of Animal Sciences (WIAS), Wageningen University, P.O. Box 338, 6700 AH Wageningen, The Netherlands

\section{J. E. Bolhuis}

Human and Animal Physiology Group,

Wageningen Institute of Animal Sciences (WIAS),

Wageningen University, P.O. Box 338,

$6700 \mathrm{AH}$ Wageningen, The Netherlands

J. E. Bolhuis $(\bowtie)$

Adaptation Physiology Group, Wageningen Institute of Animal

Sciences (WIAS), Wageningen University, P.O. Box 338,

$6700 \mathrm{AH}$ Wageningen, The Netherlands

e-mail: Liesbeth.Bolhuis@wur.nl

Present Address:

J. Jansen

Developmental Psychology, Behavioural Science Institute, Radboud University Nijmegen, P.O. Box 9104,

6500 HE Nijmegen, The Netherlands

\section{B. M. Spruijt}

Department of Biology, Utrecht University,

Padualaan 14, 3584 CH Utrecht, The Netherlands

\section{M. Wiegant}

Department of Neuroscience and Pharmacology,

Rudolf Magnus Institute of Neuroscience,

University Medical Center Utrecht,

Universiteitsweg 100, 3584 CG Utrecht, The Netherlands postpartum. Based on their escape behaviour in this test, which has been shown to reflect their behavioural style, six 'high-resisting'(HR) and six 'low-resisting' (LR) pigs were selected from each housing environment ( $n=24$ in total). At 12 weeks of age, pairs of pen mates (LR and HR) were exposed to a maze three times (exploration trials). Pigs were then placed individually in the maze, and social reinstatement proved to be a strong incentive to find the exit leading to the home pen. We subsequently blocked the direct route to the exit, forcing animals to find a detour (memory test 1, MT1). This test was repeated once to investigate the relative improvement, i.e. detour learning (memory test 2, MT2). Housing condition and Backtest response strongly affected exploration patterns. In spite of this, no effects on performance during the subsequent memory tests were found. Performance was substantially improved in MT2, indicating that once a goal is apparent, pigs are able to solve a complex spatial memory task easily. In conclusion, social reinstatement provided a good incentive to complete a spatial task, and the substantial improvement in performance between MT1 and MT2 stresses the need for task complexity when testing spatial memory in pigs. Housing conditions or individual behavioural style did not affect spatial memory during MT1 or MT2. However, housing environment and behavioural style strongly affected explorative behaviour of pigs in an unfamiliar maze during both exploration trials and memory tests. This implicates that apparent effects of environmental enrichment on spatial learning and memory in pigs might reflect differences in explorative patterns rather than in cognitive processes.

Keywords Environmental enrichment .

Behavioural style $\cdot$ Spatial learning $\cdot$ Exploration .

Social reinstatement 


\section{Introduction}

Despite a long history of domestication, the behavioural abilities of domestic pigs still closely resemble that of their wild conspecifics (Jensen 1986; Newberry and Wood-Gush 1988; Petersen 1994). Wild boars and feral pigs forage in large home ranges in which they can exploit an enormous variety of food sources (Gundlach 1986; Schley and Roper 2003). Under such conditions, individuals would benefit from good spatial memory skills and a high flexibility in behaviour (Held et al. 2005). Indeed, studies on cognitive abilities in domestic pigs have demonstrated good spatial memory skills (e.g. Mendl et al. 1997b; Laughlin et al. 1999; Laughlin and Mendl 2000).

In commercial pig husbandry, however, growing pigs are frequently housed in rather 'barren', stimulus-poor environments that may impose constraints on the development and expression of normal species-specific behaviour (Schouten 1986; Haskell et al. 1996; Wemelsfelder et al. 2000) and, possibly, cognitive abilities (De Jong et al. 2000). Particularly the lack of a suitable substrate as an outlet for the performance of exploratory activities, such as nosing, rooting and chewing, negatively affects pig behaviour. For instance, play behaviour, which may contribute to the development of adaptive cognitive skills and behavioural flexibility (Lewis 2000; Spinka et al. 2001), was observed three times less in barren-housed than in enriched-housed pigs (Bolhuis et al. 2005b).

Studies in rodents clearly indicate that stimulus-poor environments crucially affect the development of brain and behaviour, which is reflected in cognitive abilities (see Rosenzweig and Bennett 1996 for review). For instance, rearing environments lacking sufficient stimulation negatively affect behavioural flexibility (e.g. Morgan 1973; Renner and Rozenzweig 1987). Conversely, enrichment has been shown to improve the performance of rats in spatial cognitive tasks (Williams et al. 2001; Schrijver et al. 2002; Frick and Fernandez 2003; Leggio et al. 2005). Various authors have suggested that these effects of environmental enrichment on spatial memory may be mediated through alterations in hippocampal functioning, promoting hippocampal neurogenesis and dendritic branching and reducing apoptosis (Kempermann et al. 1997; Young et al. 1999; Meshi et al. 2006; Bindu et al. 2007; see also Olson et al. 2006 for a review).

Previous findings indicate changes in functional activity of the hippocampus of pigs housed in barren conditions as compared to pigs from enriched housing conditions (Van der Beek et al. 2000, Van der Beek et al., unpublished data). Studies addressing the effects of housing conditions on spatial learning and memory in pigs are, however, rather scarce and have yielded ambiguous results. Sneddon et al. (2000) found that pigs reared in an enriched environment learned a maze task faster than those reared in barren conditions. De Jong et al. (2000), however, did not find an effect of environmental conditions on the acquisition of a spatial task in pigs, although enrichment did improve long-term spatial memory in their study. This inconsistency between both studies might partly reflect differences in the complexity of the spatial task chosen rather than the impact of the housing environment, since a relatively simple maze may be unable to draw out any effect of treatment.

The impact of environmental conditions may, however, also depend on individual characteristics of the animals under study. Studies in a growing number of species describe the existence of diverging reaction patterns, sometimes also referred to as 'coping styles', 'coping strategies' or 'behavioural profiles', that resemble aspects of human personality (e.g. birds: Groothuis and Carere 2005; mammals: Koolhaas et al. 2001). The extreme phenotypes within a population differ fundamentally in the extent to which their behaviour is controlled by actual environmental stimuli (feedback control, see Koolhaas et al. 2001). Individuals at one end of the distribution are rather bold in approaching novel stimuli, and have a high tendency to develop routine-like behaviour patterns that are relatively independent of actual information. Individuals at the other end, in contrast, show more caution in making decisions, monitor even a relatively familiar environment extensively, and are therefore able to flexibly adjust their behaviour to subtle environmental changes (see Benus et al. 1991; Koolhaas et al. 2001 for review).

It has been suggested that the behavioural profile of pigs can be assessed early in life on the basis of their response in a Backtest (Hessing et al. 1993; Geverink et al. 2003; Bolhuis 2004). In the Backtest, piglets are manually restrained in supine position for $1 \mathrm{~min}$ and their behavioural response, which ranges from vigorous struggling to complete immobility, is recorded (Hessing et al. 1993; Bolhuis et al. 2004). Pigs that struggle most in the test, the 'high resisters' (HR), have been reported to explore a novel stimulus rather fast and superficially, whereas 'low-resisters' (LR) were suggested to explore more slowly and thoroughly (Hessing et al. 1994; Ruis et al. 2000, 2001), but these results were all found during a single exposure to novelty. The two 'types' of pig also differ in the ability to adjust their behaviour to a changing situation (Geverink et al. 2004; Bolhuis et al. 2004, 2005a). For instance, HR pigs were less successful in reversal learning in a T-maze task than LR pigs, suggesting that they have a higher propensity to develop inflexible behavioural routines that are difficult to inhibit under changing circumstances. In contrast, both in a social and non-social context, LR pigs flexibly altered their behaviour patterns when appropriate (Bolhuis et al. 2004; 2005a). Moreover, the impact of a barren environment as opposed to enriched housing differed between LR and HR 
pigs, with larger effects of environmental conditions on behaviour and physiology in LR pigs (Bolhuis et al. 2003, 2004, 2005b, 2006). Hence, the two 'types' of pig at either end of the distribution differ in their response to a wide range of challenging conditions later in life (Bolhuis et al. 2004; Geverink et al. 2003; Hessing et al. 1994; Ruis et al. 2000)

The aim of the present study was to investigate the influence of both environmental conditions during rearing (barren vs. enriched) and individual behavioural characteristics (HR vs. LR) on the behavioural response to repeated exposures to a novel environment and on spatial cognitive capabilities in pigs. To that end, we used a novel spatial learning paradigm. The paradigm is based on Tolman and Honzik's detour problem described in Tolman (1948), which has been applied in several species (e.g. cats: Poucet et al. 1983; dogs: Chapuis 1987; birds: Regolin et al. 1994, 1995; Zucca et al. 2005; quokkas: Wynne and Leguet 2004), using various adaptations of the detour maze. The detour task is characterised by the necessity to avoid a direct route to a goal, but instead find an alternative route. Tolman proposed that animals utilize a cognitive map of the environment to do so, and O'Keefe and Nadel (1978) later suggested the hippocampus as the seat of this cognitive map.

The effects of housing environment and behavioural characteristics on the response to repeated exposures to novelty, and subsequent effects on latent and goal-directed detour learning, have not been assessed in pigs. In addition, the use of social reinstatement rather than food deprivation as the incentive to complete a task has been proposed (Wickert and Barr 1966, cited by Hammell et al. 1975), but not been utilized in more recent studies on spatial memory.

We firstly exposed pigs to a novel, complex maze environment in a series of exploration trials. We chose to subject pairs of pen mates to the maze, because social isolation is known to induce stress in group-housed pigs (e.g. Ruis et al. 2001), which in turn might affect their behavioural response. After the exploration trials, we tested (latent) spatial memory in the pigs by exposing them to the maze individually, this time using social reinstatement as an incentive to find the exit leading to the home pen. To complicate the test, we subsequently blocked the direct route to the exit, forcing animals to find a detour to reach it [memory test 1 (MT1)]. In this detour test, pigs had to move away from the exit initially in order to reach it, comparable to the detour tasks presented in Regolin et al. $(1994,1995)$. Detour behaviour in this first memory test should require maintenance of a cognitive map of the maze, and the ability to flexibly adjust behaviour (Chapuis 1987; Zucca et al. 2005). This test was repeated once to investigate the relative improvement in pigs with different housing background and Backtest classification, i.e. detour learning [memory test 2 (MT2)]. Pigs from barren housing were expected to perform worse than those from enriched home environments. In addition, we hypothesised that LR pigs would explore the maze more thoroughly than HR pigs, and consequently would perform better in the memory tests, and that the effects of housing would be most profound in LR pigs.

\section{Methods}

All experimental procedures were carried out at the experimental farm of Wageningen University in 2001. The experiment was carried out in two batches with 12 Great Yorkshire $\times($ Great Yorkshire $\times$ Dutch Landrace) barrows each.

Pre-weaning housing conditions

Experimental animals were offspring of 21 sows per batch that had been purchased for the breeding of piglets for another experiment (Bolhuis et al. 2005b). One week prior to the expected date of farrowing, sows were housed in farrowing pens with removable crates $(2.2 \times 3.8 \mathrm{~m}, 65 \%$ solid and $35 \%$ slatted floor). Half of the sows were provided with approximately $4 \mathrm{~kg}$ of unchopped straw bedding material (enriched housing; E), while the remaining sows were housed on barren flooring (barren housing; B).

One day after birth of the experimental animals, tails were docked, ear tattoos were applied and an iron injection was administered following standard husbandry procedures. All males were castrated three days after birth. Water was available from a nipple drinker in the pen and from 10 days after birth creep feed was provided in the form of prestarter concentrate. Lights were on between 7.00 and $19.00 \mathrm{~h}$. All pens were cleaned between 8.00 and $9.00 \mathrm{~h}$ and pigs housed under enriched conditions were then provided fresh unchopped straw.

\section{Backtest and selection of animals}

All pigs were subjected to a Backtest adapted from Hessing et al. (1993) at 10 and 17 days of age. The test was carried out on a table placed in a room near the home pen. The experimenter lifted a piglet and used the left hand to place the piglet on its back on a $25 \mathrm{~kg}$-feed bag $(1 \times \mathrm{b} \times \mathrm{h} 72 \times$ $52 \times 13 \mathrm{~cm}$ ) covered with plastic. The right hand of the experimenter was then placed firmly on the thorax of the piglet, with the left foreleg of the piglet between the experimenter's thumb and index finger, and the right foreleg between index and middle finger. The experimenter's left hand was used to stretch and move the hind legs of the piglet downward, after which the test started. Pigs were 
restrained in this supine position for $60 \mathrm{~s}$. During the test, the experimenter's left hand remained loosely on the hind legs of the piglet, and was used only to maintain the piglet's supine position during and after struggling with the hind legs. Each bout of struggling with at least the hind legs was counted as one escape attempt. The number of escape attempts was used as the basis for classifying high resisting (HR; $\geq 3+\geq 2$ escape attempts during the first and second Backtest) and low resisting (LR; $\leq 2+\leq 1$ escape attempts) animals (see Hessing et al. 1994). Twenty-four healthy barrows were randomly selected from the pool of LR and HR pigs (six barren HR, six enriched HR, six barren LR and six enriched LR) for the experiment. The selected animals were given a plastic ear tag with an individual number.

\section{Post-weaning housing conditions}

At 4 weeks of age, experimental animals were weaned and mixed. Two experimental barrows (one LR and HR) were placed in standard commercial pens (approx. $7 \mathrm{~m}^{2}, 65 \%$ concrete flooring, $35 \%$ slats) together with four gilts (two HR and two LR, selected from the same pool of pigs). All animals (gilts and barrows) were used for another observational study (Bolhuis et al. 2005b), but only the barrows were subjected to the current experiment. Prior to mixing, all animals were unfamiliar with each other.

Animals from barren farrowing pens (B pigs) were housed in barren pens. Animals reared under enriched conditions (E pigs) were placed in identical pens, but were provided with unchopped straw bedding material (approximately $4 \mathrm{~kg}$ per pen). In the $\mathrm{E}$ pens, a rubber mat covered the slatted area to prevent leakage of straw. The pigs were fed a standard commercial diet ad libitum. Two nipple drinkers per pen provided water. The pens were cleaned and fresh straw was provided each morning between 08.00 and $09.00 \mathrm{~h}$. Lights were on between 07.00 and $19.00 \mathrm{~h}$ throughout the experiment.

Handling

At 6 weeks of age all experimental animals were handled. All persons taking part in later testing spent $10 \mathrm{~min}$ in each pen, allowing piglets to touch them to get accustomed to their voices and odour. At 7 weeks of age all animals were taken out of the pen and habituated to the corridor adjacent to their home pens and the waiting area of the adjoining room (where subsequent tests were carried out). The animals were not exposed to the testing arena itself.

\section{Exploration trials}

The maze is shown in Fig. 1. The maze, located in the room next to the home pens, was constructed of dark brown

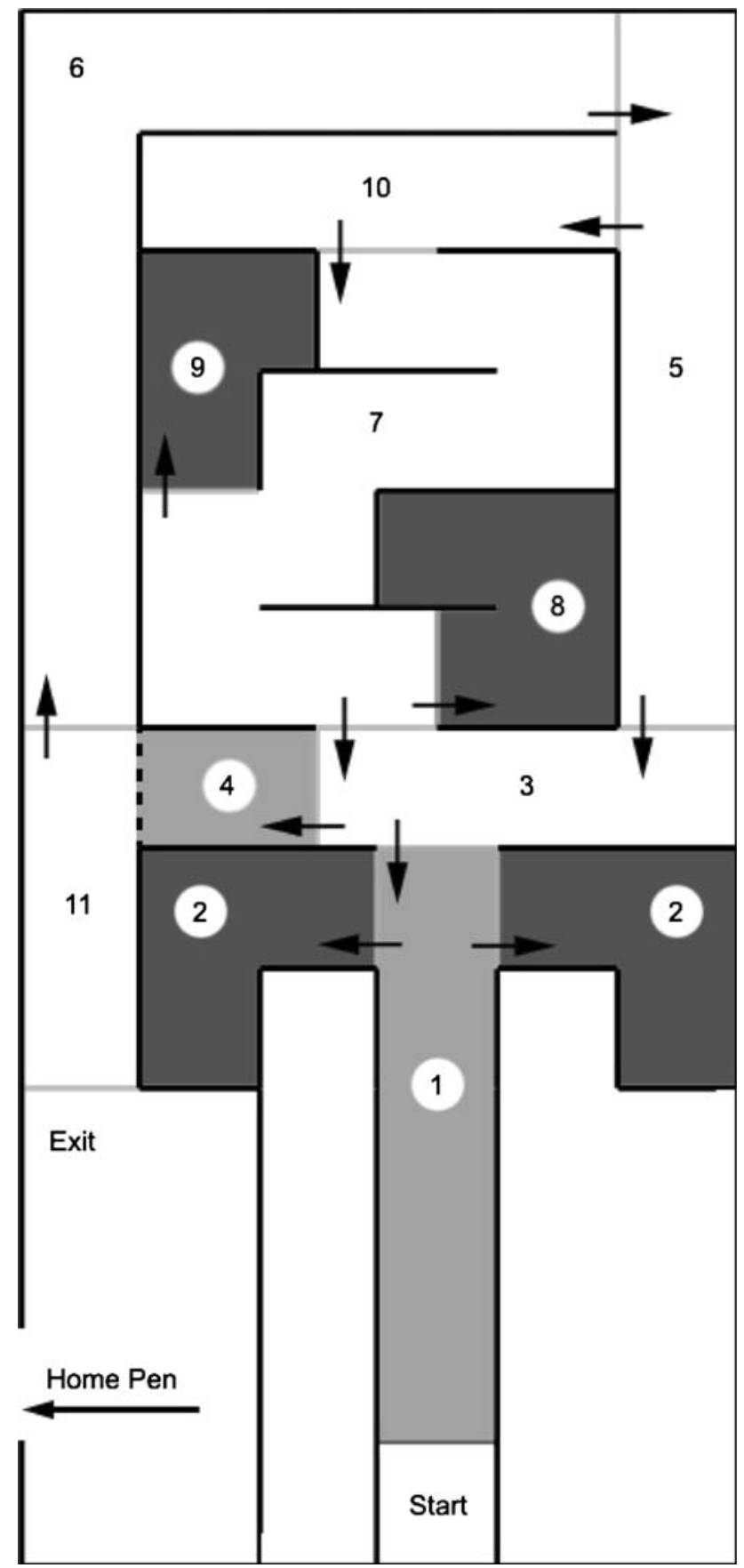

Fig. 1 Maze. Numbers indicate sections, light grey lines denote imaginary borders, small arrows indicate erroneous crossings during memory tests. Dark grey sections indicate Wrong sections, light grey sections denote Persistent sections. Dashed line indicates fence (open during exploration, closed during memory tests)

wooden walls, and measured $6 \times 13 \mathrm{~m}$ in total, with corridors of approximately $1 \mathrm{~m}$ in width. The maze was divided into 12 sections by imaginary lines and contained a starting box and an exit that led to the home pens of the pigs. At 12 weeks of age, all animals were placed in the maze on three consecutive days (exploration trial 1,2 and 3) for 720, 720 and $600 \mathrm{~s}$, respectively. Animals were subjected to the 
maze in pairs of pen mates (one HR and one LR barrow) to avoid stress caused by social isolation (e.g. Ruis et al. 2001). No food rewards were hidden anywhere in the maze. In each exploration trial the animals were led to a different starting point (sections 1, 6, and 9, respectively) to prevent formation of exploration habits and promote thorough exploration of the arena.

\section{Memory tests}

Two days after the last exploration trial, animals were individually placed in the starting box of the maze to habituate them to an individual exposure to the maze and to test whether they were able to reach the exit (and their home pen) within $30 \mathrm{~s}$. All animals accomplished this task within $30 \mathrm{~s}$, showing that they were highly motivated to return to their home pen. Social reinstatement has previously been successfully used as an incentive to complete a task (in pigs: Wickert and Barr 1966, cited by Hammell et al. 1975, in chicks: Vallortigara et al. 1990). All animals took the shortest route (through sections 4 and 11; see Fig. 1) to reach the exit.

On the subsequent day, the first memory test (MT1) was carried out. A fence was placed between Sect. 4 and 11, blocking the shortest route to the exit (Fig. 1). All animals were again individually placed in the starting box of the maze and now had to find the exit via an alternative route. If the animal had not reached the exit within $15 \mathrm{~min}$, it was led back to the exit via sections $3,5,6$, and 11 and given the maximum time score.

The day after the first memory test, all individual barrows were placed into the starting box of the maze again (memory test 2) to assess whether the previous individual exposure had positively affected their performance. If the animal had not reached the exit within $15 \mathrm{~min}$, it was led back to the exit via sections $3,5,6$, and 11 and given the maximum time score. In the results section, the first and second memory tests are referred to as MT1 and MT2, respectively.

\section{Observations}

During all maze exposures, behaviour of the individual pigs was scored continuously by two observers sitting near the maze in elevated chairs, using The Observer 3.0 software package (Noldus Information Technology B.V., Wageningen, The Netherlands) installed on a Psion organiser. Individual pigs could be identified by a number marked on their backs. In order to characterize the explorative pattern of pigs during the exploration trials, the posture of the animal and its behaviour were recorded continuously, using the following ethogram with two classes: Postures and locomotion: standing, walking, running (i.e., trotting or gallop- ing), lying or sitting on hind limbs; Other behavioural activities: standing alert (standing immobile with open eyes, raised head and pricked ears), nosing (keeping rooting disc at or close to the floor or walls) or other. Vocalisations were scored as events.

In addition, the location in the maze (section number) was continuously scored. An animal entered a new section when it had crossed the imaginary line with four legs. From these recordings, the total number of line crossings was calculated, as well as the time spent in and latencies to enter the different sections.

To evaluate the performance of pigs during MT1 and MT2, the number of errors, defined as line crossings that could not lead to the exit, and the latency to reach the exit were also scored. Percentages of time spent in sections 2, 8 and 9 were combined and defined as Wrong [presence in these sections would never lead to the exit (e.g. Regolin et al. 1995)]; presence in sections 1 or 4 was labelled Persistent (sections 1 and 4 could lead back to the home-pen in the exploration trials and individual habituation trial, but not in MT1 and MT2).

Data analysis

Skewed distributions of frequency, duration and proportion were square root, logarithmically and arcsine square root transformed, respectively, to obtain homogeneity of variance.

Effects of housing condition (barren vs. enriched), Backtest classification (HR vs. LR) and their interaction on the latencies to enter new sections in trial 1, 2 and 3 were analysed with a mixed linear model allowing for random effects of pen and batch.

Effects of housing condition (barren vs. enriched), Backtest classification (HR vs. LR), trial (1, 2 or 3) and their interactions on behaviour during exploration trials were analysed with a mixed linear model allowing for random effects of animal, pen and batch. Running and walking were combined and analysed as a single behavioural element: 'locomotion'. The behavioural elements 'lying' and 'sitting' were excluded from analysis because they hardly occurred (means over the three exploration trials: HR-B: $5.9 \pm 5.5 \%$; LR-B: $3.3 \pm 2.7 \%$; HR-E: $0.2 \pm 0.2 \%$; LR-E: $0.8 \pm 0.8 \%)$. As a result, effects on standing and locomotion (adding to almost $100 \%$ ) were very similar; therefore only effects on locomotion are presented in the results section.

Effects of housing and Backtest classification on behaviour and performance in MT1 and MT2 were analysed with a mixed linear model allowing for random effects of pen and batch. Changes in performance in MT2 as compared to MT1 were analysed by including a trial effect (MT1 vs. MT2) and its interactions with housing and Backtest 
classification in the model, and the random effects of animal. For brevity, only significant interactions with trial (MT1 vs. MT2) are reported in the results section.

Significant interaction effects were further analysed using post hoc tests with Tukey adjustments for multiple comparisons. All data are presented as mean \pm SEM. Due to technical difficulties, we were unable to score behavioural data for one animal during the first exploration trial, but we did record the number of vocalisations on paper.

\section{Results}

\section{Exploration trials}

\section{Location in the maze and line crossings}

Latency to enter new sections. HR pigs were faster than LR pigs to enter a first $\left(F_{1,9}=6.9, P=0.027\right)$, third $\left(F_{1,9}=6.1\right.$, $P=0.036)$ and fourth $\left(F_{1,9}=7.0, P=0.027\right)$ novel section (Fig. 2, latency to enter second novel section $\left(F_{1,9}=4.8\right.$, $P=0.057)$ ) in trial 1, but they did not differ from LR pigs in entering sections during trial 2 and 3 . Housing condition did not affect latencies to enter new sections in any trial (data of trials 2 and 3 not shown).

Line crossings. Pigs from enriched housing (E pigs) crossed more lines per minute $(4.7 \pm 0.4)$ than barrenhoused (B) pigs $(3.2 \pm 0.1)$ across all three trials $\left(F_{1,9}=15.2, P=0.004\right)$. Backtest classification did not affect the number of line crossings $\left(F_{1,10}=0.31, P=0.59\right.$; HR: $4.0 \pm 0.3$, LR: $3.9 \pm 0.3$ ). The effect of exploration trial on the number of line crossings per minute was not significant $\left(F_{2,39}=2.9, P=0.069\right)$.

\section{Locomotion and behaviour}

Locomotion. Locomotion was affected by housing condition $\left(F_{1,9}=12.8, P=0.006\right.$; Fig. 3$)$, trial $\left(F_{2,39}=7.4, P=0.002\right)$ and the interaction between Backtest classification and trial $\left(F_{2,39}=4.5, P=0.018\right)$. Locomotion was rather constant

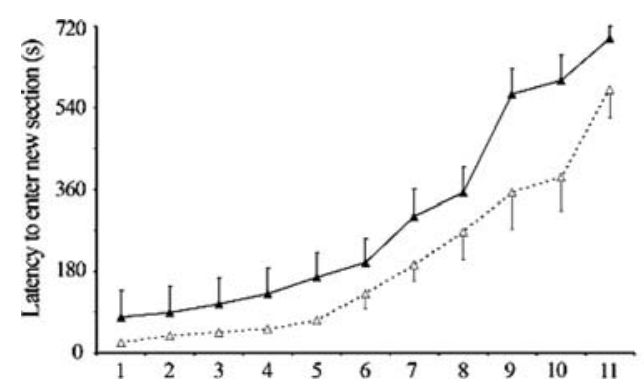

Fig. 2 Effects of housing (left panel) and Backtest classification (right panel) on the cumulative number of new sections visited during exploration trial 1. Closed triangles, solid lines barren housing; open trian- over trials for LR pigs, whereas HR pigs spent more time on locomotion during the first trial as compared to the other trials (post hoc Tukey test, $P<0.05$; Fig. 3).

Nosing. Nosing was affected by housing condition $\left(F_{1,9}=36.6, P<0.001\right)$, trial $\left(F_{2,39}=7.8, P=0.001\right)$. E pigs, but not $\mathrm{B}$ pigs, tended to show a decrease in nosing over trials (Fig. 3); however, the housing $\times$ trial interaction was not significant $\left(F_{2,39}=3.0, P=0.060\right)$. LR pigs spent more time nosing than $\mathrm{HR}$ pigs during exploration trials (Backtest classification effect, $F_{1,10}=6.9, P=0.025$ ).

Standing alert. E pigs spent more time on standing alert than B pigs $\left(F_{1,9}=17.4, P=0.002 ;\right.$ Fig. 4$)$. Standing alert increased with trial in LR pigs, but decreased in HR pigs (Backtest classification $\times$ trial interaction, $F_{2,39}=6.9$, $P=0.004$; Fig. 4).

Vocalisations. Vocalisation frequency was affected by housing condition $\left(F_{1,9}=24.4, P<0.001\right)$ and the housing condition $\times$ trial interaction $\quad\left(F_{2,39}=5.8, \quad P<0.01\right.$, $P=0.007)$. E pigs showed an increase in vocalisations over trials, whereas B pigs showed a decrease (Fig. 4). HR pigs vocalised more often than LR pigs during exploration trials, $\left(F_{1,10}=7.8, P=0.019\right.$; Fig. 4$)$.

\section{Memory test 1 (MT1)}

\section{Performance and line crossings (Table 1)}

Latency to reach the exit. Latency to reach the exit in MT1 ranged from 101 to $900 \mathrm{~s}$. Seven animals had to be guided to the exit: five B pigs (three HR and two LR) and two E pigs (two LR). Housing condition $\left(F_{1,9}=0.53, P=0.48\right.$ ), Backtest classification $\left(F_{1,10}=0.51, P=0.49\right)$ or their interaction $\left(F_{1,10}=1.1, P=0.33\right)$ did not affect the latency to reach the exit in MT1.

Number of errors. The number of errors ranged from 4 to 39 and was not affected by housing condition $\left(F_{1,9}=0.00, P=0.99\right)$, Backtest classification $\left(F_{1,10}=0.08\right.$, $P=0.79)$ or their interaction $\left(F_{1,10}=2.1, P=0.18\right)$.

Time spent in Wrong and Persistent sections. Housing condition $\left(F_{1,9}=0.40, P=0.54\right)$ and Backtest classification

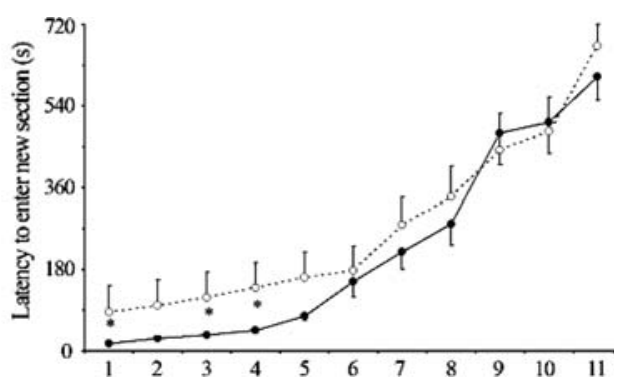

gles, dotted lines enriched housing (left panel). Closed circles, solid lines HR, open circles, dotted lines LR (right panel) 
Fig. 3 Effects of housing (left panels) and Backtest classification (right panels) on percentages of time spent on locomotion and nosing. Closed triangles, solid lines barren housing; open triangles, dotted lines enriched housing (left panels). Closed circles, solid lines HR; open circles, dotted lines LR (right panels)

Fig. 4 Effects of housing (left panels) and Backtest classification (right panels) on the percentage of time spent on standing alert and the number of vocalisations per minute. Closed triangles, solid lines barren housing; open triangles, dotted lines enriched housing (left panels). Closed circles, solid lines HR; open circles, dotted lines LR (right panels)
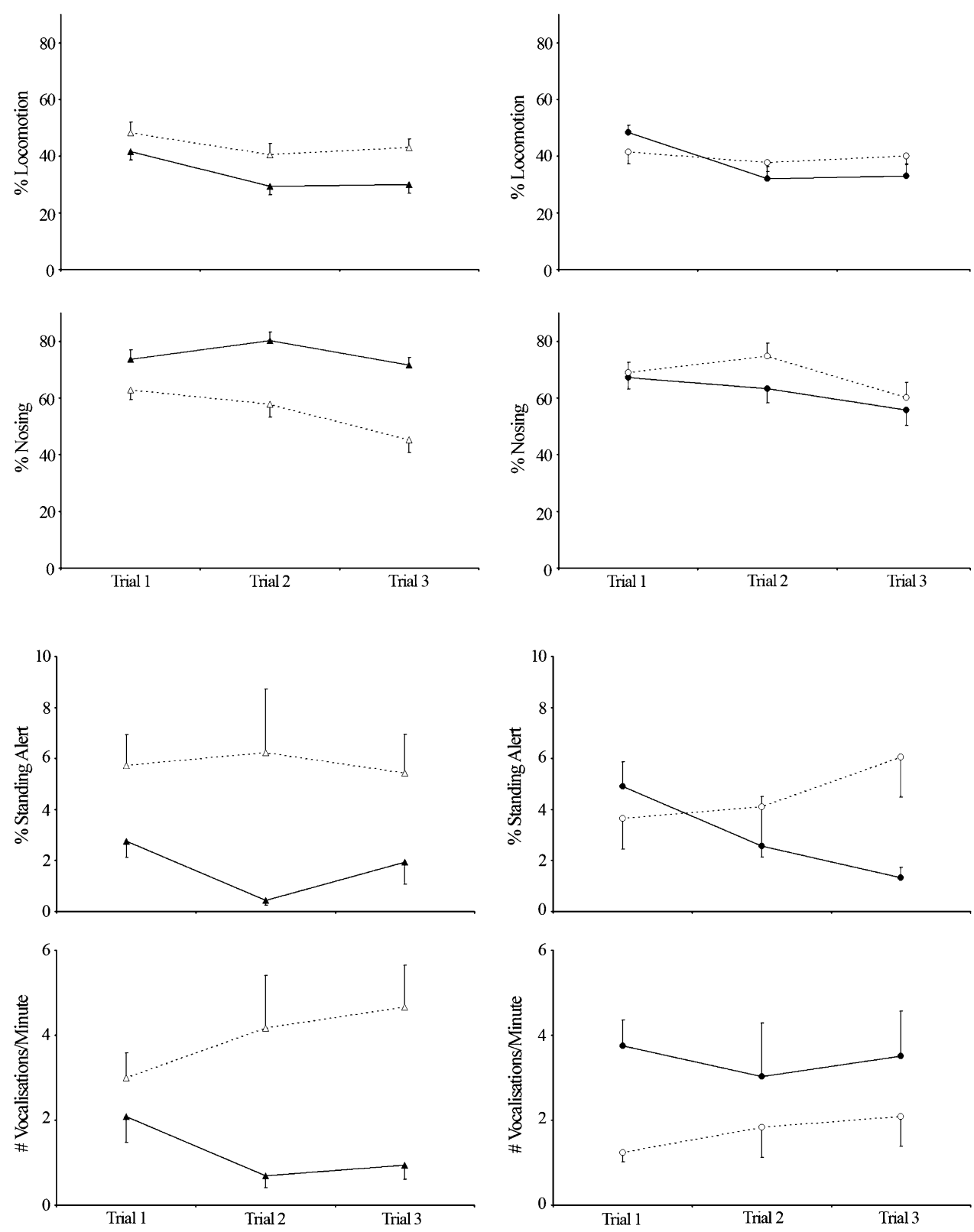

$\left(F_{1,10}=0.23, P=0.64\right)$ did not affect the percentage of time spent in Wrong sections. B pigs spent less time in Persistent sections than E pigs $\left(F_{1,9}=15.9, P=0.025\right)$. LR pigs spent less time in Persistent sections than HR pigs $\left(F_{1,10}=8.7\right.$, $P=0.015)$.

Line crossings. Housing condition $\left(F_{1,9}=3.0, P=0.14\right)$, Backtest classification $\left(F_{1,10}=0.15, P=0.71\right)$ or their interaction $\left(F_{1,10}=0.00, P=0.96\right)$ did not affect the number of line crossings per minute.

\section{Locomotion and behaviour (Table 2)}

Locomotion. Housing condition $\left(F_{1,9}=0.63, P=0.45\right)$, Backtest classification $\left(F_{1,10}=1.8, P=0.21\right)$ and their interaction $\left(F_{1,10}=0.02, P=0.89\right)$ did not affect the time spent on locomotion.

Nosing. B pigs nosed more than E pigs during MT1 $\left(F_{1,9}=14.7, P=0.004\right)$. No effects of Backtest classification $\left(F_{1,10}=0.65, P=0.44\right)$ or its interaction with housing $\left(F_{1,10}=0.01, P=0.93\right)$ were found.

Standing alert. Housing condition $\left(F_{1,9}=0.30\right.$, $P=0.36)$, Backtest classification $\left(F_{1,10}=0.30, P=0.60\right)$ and their interaction $\left(F_{1,10}=3.3, P=0.10\right)$ did not affect time spent standing alert (Table 2).

Vocalisations. HR pigs vocalised more than LR pigs $\left(F_{1,10}=5.0, P=0.049\right)$. No effect of housing $\left(F_{1,9}=4.3\right.$, $P=0.07)$ or the housing $\times$ Backtest classification interaction was found $\left(F_{1,10}=0.01, P=0.94\right)$. 
Table 1 Performance and location in the maze of LR and HR pigs from barren or enriched housing during MT1 and MT2

\begin{tabular}{|c|c|c|c|c|c|c|c|c|c|c|c|c|c|c|c|c|}
\hline & \multicolumn{8}{|l|}{ MT1 } & \multicolumn{8}{|l|}{ MT2 } \\
\hline & \multicolumn{4}{|c|}{ Barren housing } & \multicolumn{4}{|c|}{ Enriched housing } & \multicolumn{4}{|c|}{ Barren housing } & \multicolumn{4}{|c|}{ Enriched housing } \\
\hline & \multicolumn{2}{|l|}{ HR } & \multicolumn{2}{|l|}{ LR } & \multicolumn{2}{|l|}{ HR } & \multicolumn{2}{|l|}{ LR } & \multicolumn{2}{|l|}{ HR } & \multicolumn{2}{|l|}{ LR } & \multicolumn{2}{|l|}{ HR } & \multicolumn{2}{|l|}{ LR } \\
\hline & $M$ & SE & $M$ & SE & $M$ & SE & $M$ & SE & $M$ & SE & $M$ & SE & $M$ & SE & $M$ & SE \\
\hline Lat exit (s) & 664.8 & 122.0 & 545.7 & 143.6 & 443.5 & 131.2 & 526.5 & 162.1 & 160.5 & 33.2 & 112.7 & 37.1 & 49.0 & 6.7 & 113.3 & 41.9 \\
\hline Errors (no.) & 22.2 & 3.8 & 14.7 & 3.5 & 19.7 & 4.2 & 22.8 & 6.1 & 3.5 & 0.8 & 2.7 & 1.0 & 2.3 & 0.7 & 3.5 & 1.5 \\
\hline Incorr (\%) & 51.4 & 6.1 & 40.5 & 8.1 & 643 & 1.6 & 51.8 & 5.0 & 41.4 & 9.7 & 33.7 & 4.9 & 32.6 & 4.4 & 33.5 & 8.3 \\
\hline Wrong (\%) & 16.8 & 3.0 & 15.1 & 6.7 & 11.4 & 3.7 & 13.3 & 4.3 & 15.4 & 6.9 & 11.8 & 5.1 & 3.9 & 2.8 & 3.3 & 3.3 \\
\hline Persist (\%) & 34.6 & 4.9 & 25.3 & 3.5 & 52.9 & 3.2 & 38.4 & 3.9 & 26.0 & 6.4 & 21.9 & 2.8 & 28.7 & 4.1 & 30.3 & 6.3 \\
\hline $\mathrm{Cr} / \min$ (no.) & 5.7 & 1.4 & 5.1 & 1.1 & 7.9 & 1.8 & 7.3 & 1.2 & 6.9 & 1.8 & 9.3 & 2.3 & 14.7 & 2.0 & 9.5 & 1.5 \\
\hline
\end{tabular}

Lat exit latency to reach the exit, Incorr, Wrong, Persistent percentages of time spent in Incorrect, Wrong and Persistent sections of the maze, $\mathrm{Cr} / \mathrm{min}$ number of line crossings per minute. Significant effects are described in the text

Table 2 Behaviour of LR and HR pigs from barren or enriched housing during MT1 and MT2

\begin{tabular}{|c|c|c|c|c|c|c|c|c|c|c|c|c|c|c|c|c|}
\hline & \multicolumn{8}{|l|}{ MT1 } & \multicolumn{8}{|c|}{ MT2 } \\
\hline & \multicolumn{4}{|c|}{ Barren housing } & \multicolumn{4}{|c|}{ Enriched housing } & \multicolumn{4}{|c|}{ Barren housing } & \multicolumn{4}{|c|}{ Enriched housing } \\
\hline & \multicolumn{2}{|l|}{ HR } & \multicolumn{2}{|l|}{ LR } & \multicolumn{2}{|l|}{ HR } & \multicolumn{2}{|l|}{ LR } & \multicolumn{2}{|l|}{ HR } & \multicolumn{2}{|l|}{ LR } & \multicolumn{2}{|l|}{ HR } & \multicolumn{2}{|l|}{ LR } \\
\hline & $M$ & SE & $M$ & SE & $M$ & SE & $M$ & SE & $M$ & SE & $M$ & SE & $M$ & SE & $M$ & SE \\
\hline $\operatorname{Loco}(\%)$ & 40.7 & 8.9 & 46.0 & 6.6 & 46.6 & 4.6 & 53.1 & 7.1 & 44.0 & 8.1 & 60.0 & 9.8 & 73.8 & 4.1 & 61.0 & 7.8 \\
\hline Nosing (\%) & 54.3 & 6.6 & 60.0 & 5.8 & 30.7 & 10.1 & 35.2 & 5.8 & 46.6 & 9.4 & 28.7 & 12.1 & 6.4 & 3.9 & 23.7 & 9.5 \\
\hline Alert (\%) & 1.3 & 0.6 & 0.5 & 0.3 & 2.4 & 1.3 & 2.9 & 1.4 & 0.1 & 0.0 & 0 & 0 & 0 & 0 & 0.1 & 0.1 \\
\hline Voc/min (no.) & 11.9 & 2.8 & 7.3 & 3.4 & 22.5 & 3.8 & 15.0 & 5.2 & 9.8 & 2.1 & 5.3 & 2.2 & 11.7 & 3.4 & 7.6 & 1.3 \\
\hline
\end{tabular}

Loco locomotion, Alert standing alert, Voc/min number of vocalisations per minute, respectively. Significant effects are described in the text

\section{Memory test 2 (MT2)}

\section{Performance and line crossings (Table 1)}

Latency to reach the exit. All animals reached the exit faster in MT2 than in MT1 $\left(F_{1,20}=119.8, P<0.001\right)$ regardless of housing $\left(F_{1,20}=0.87, P=0.36\right)$ or Backtest classification $\left(F_{1,20}=0.33, P=0.57\right)$. During MT2, no significant effects of housing $\left(F_{1,9}=4.3, P=0.07\right)$, Backtest classification $\left(F_{1,10}=0.01, P=0.92\right)$ or their interaction $\left(F_{1,10}=2.9, P=0.92\right)$ on the latency to reach the exit was found.

Number of errors. Pigs made fewer errors during MT2 than during MT1 $\left(F_{1,20}=36.0, P<0.001\right)$. This decrease was not affected by housing $\left(F_{1,20}=0.42, P=0.53\right)$ or Backtest classification $\left(F_{1,20}=0.27, P=0.61\right)$. The number of errors during MT2 was not affected by housing $\left(F_{1,9}=0.42, P=0.53\right)$, Backtest classification $\left(F_{1,10}=0.34\right.$, $P=0.57)$ or their interaction $\left(F_{1,10}=0.57, P=0.47\right)$.

Time spent in Wrong and Persistent sections. Time spent in Wrong and Persistent sections decreased $\left(F_{1,20}=8.4\right.$ and
$F_{1,20}=11.5$, respectively, $\left.P=0.009\right)$ irrespective of housing (housing $\times$ MT trial effect $F_{1,20}=2.2$ and 1.8, $P=0.15$ and 0.19, respectively) or Backtest classification (Backtest classification $\times$ MT trial effect, $F_{1,20}=0.00$ and 2.4, $P=0.95$ and 0.14 , respectively). E pigs and B pigs did not significantly differ in the percentage of time spent in Wrong sections $\left(F_{1,10}=4.7, P=0.056\right)$. Also Backtest classification $\left(F_{1,10}=0.21, P=0.65\right)$ or its interaction with housing $\left(F_{1,10}=0.01, P=0.93\right)$ did not significantly affect time spent in Wrong sections. Time spent in Persistent sections during MT2 was not affected by housing $\left(F_{1,9}=1.3\right.$, $P=0.29)$, Backtest classification $\left(F_{1,10}=0.02, P=0.87\right)$ or their interaction $\left(F_{1,10}=0.14, P=0.71\right)$.

Line crossings. The number of line crossings per minute was higher during MT2 than during MT1 $\left(F_{1,20}=31.6\right.$, $P<0.001)$. The increase was affected by the housing $\times$ Backtest classification interaction (housing $\times$ Backtest classification $\times$ MT trial effect, $F_{1,20}=7.6$, $P=0.012$ ). Post hoc analysis revealed that the E-HR pigs showed the most profound increase in the number of line crossings per minute from MT1 to MT2. 
Housing $\left(F_{1,9}=5.1, P=0.050\right.$ affected the number of line crossings in MT2. The effects of Backtest classification $\left(F_{1,10}=0.33, P=0.58\right)$, and the housing $\times$ Backtest classification interaction $\left(F_{1,10}=3.5, P=0.093\right)$ on line crossings were not significant.

\section{Locomotion and behaviour (Table 2)}

Locomotion. Percentage of time spent on locomotion increased from MT1 to MT2 $\left(F_{1,20}=23.1, P<0.001\right)$. Particularly the E-HR pigs showed an increase in locomotion from MT1 to MT2 (housing $\times$ Backtest classification $\times$ MT trial effect: $\left.F_{1,20}=7.6, P=0.012\right)$.

Effects of housing condition $\left(F_{1,9}=4.6, P=0.061\right)$, Backtest classification $\left(F_{1,10}=0.05, P=0.83\right)$ and the housing $\times$ Backtest classification interaction $\left(F_{1,10}=4.0\right.$, $P=0.073$ ) on locomotion during MT2 were not significant.

Nosing. Percentage of time spent on nosing decreased from MT1 to MT2 $\left(F_{1,20}=19.6, P<0.001\right)$. Particularly EHR and B-LR pigs showed less nosing in MT2 than in MT1 (housing $\times$ Backtest classification $\times$ MT trial effect: $\left.F_{1,20}=4.6, P=0.044\right)$.

$B$ pigs nosed relatively more than $E$ pigs during MT2 $\left(F_{1,9}=8.1, P=0.019\right)$. The housing condition $\times$ Backtest classification interaction was not significant $\left(F_{1,10}=4.9\right.$, $P=0.051)$.

Standing alert. Percentage of time spent standing alert decreased from MT1 to MT2 $\left(F_{1,20}=24.6, P<0.001\right)$, irrespective of housing condition or Backtest classification.

Housing condition $\left(F_{1,9}=0.01, P=0.91\right)$, Backtest classification $\left(F_{1,10}=0.01, P=0.91\right)$ or their interaction $\left(F_{1,10}=2.0, P=0.19\right)$ did not affect standing alert during MT2.

Vocalisations. The number of vocalisations was lower during MT2 than during MT1 $\left(F_{1,20}=8.2, P=0.010\right)$. The housing condition $\times$ MT trial $\left(F_{1,20}=3.3, P=0.084\right)$ and Backtest classification $\times$ MT trial effects were not significant $\left(F_{1,20}=0.58, P=0.46\right)$.

HR pigs did not vocalise more than LRs during MT2 $\left(F_{1,10}=5.0, \quad P=0.050\right)$. Housing condition $\left(F_{1,9}=2.1\right.$, $P=0.18)$ and its interaction with Backtest classification $\left(F_{1,10}=0.35, P=0.57\right)$ did not affect the number of vocalisations per minute.

\section{Discussion}

Explorative behaviour and spatial memory were tested in a novel detour paradigm in pigs differing in housing environment during rearing (B vs. E), and in Backtest classification (HR vs. LR). Behaviour during exploration trials was affected by housing and Backtest classification. In spite of obvious effects on behaviour during the exploration trials, we found no strong effects of housing or Backtest classification on performance during the memory tests. We did find, however, that animals greatly improved in their performance between successive memory tests and that social reinstatement proved to be a strong incentive to complete the task.

\section{Exploration trials}

During exploration trials the pigs could easily be led to the starting area in the maze, indicating that the procedure was not stressful to the animals. After the first exploration, most pigs, when let out of their home pens, ran to the maze. Both housing condition and Backtest classification largely influenced behaviour patterns during exploration trials.

Effects of housing conditions. B pigs showed more nosing behaviour during exploration trials than E pigs. This agrees with other studies reporting an increased exploration of novel environments or objects in pigs from barren housing, and has been attributed to an unsatisfied motivation for exploration in their home environment (Mendl et al. 1997a; De Jong et al. 1998; Olsson et al. 1999; Bolhuis 2004).

In contrast, $\mathrm{E}$ pigs spent more time on locomotion than $\mathrm{B}$ pigs during exploration trials. Higher levels of activity following exposure to a novel environment has also been reported in another study on pigs (Wemelsfelder et al. 2000) and in studies on rats (Zimmermann et al. 2001) and chickens (Freire et al. 2004) reared in relatively complex environments. Wemelsfelder et al. (2000) suggested that enrichment affected the pigs' tendency to explore and interact with the environment. However, given our observation that B pigs showed more nosing of the novel maze, it is also possible that enrichment, rather than altering the motivation to explore, changes explorative behavioural patterns of animals. Although E pigs were provided with a rooting substrate in their home environment, the size of the E pens was equal to that of the B pens. The larger amount of locomotive behaviour of enriched pigs might thus reflect that only if 'nosing needs' have been fulfilled in the home environment, 'locomotion needs' are expressed. Alternatively, the higher levels of locomotion, in combination with higher levels of vocalising and standing alert, may have reflected increased fearfulness (cf. Beattie et al. 2000) or vigilance in E pigs as compared to B pigs. This striking difference in explorative patterns between barren and enriched housed pigs was also reflected in behaviour during MT1. Inconsistent effects of environmental enrichment on spatial learning and memory in previous studies (De Jong et al. 2000; Sneddon et al. 2000) might therefore partly be explained by effects of environmental enrichment on explorative behaviour. For instance, Sneddon et al. (2000) focused on time as a measure of spatial cognitive performance and found the faster exploring $\mathrm{E}$ pigs doing better, whereas others (De 
Jong et al. 2000) focused on the number of errors and found no effects of environmental enrichment. Studies on the effects of environmental enrichment on spatial cognition should therefore account for the possibility that apparent differences in memory or learning might result from differences in explorative style rather than in cognitive processes.

Effects of Backtest classification. During the first exploration trial, HR animals showed more locomotion than LR animals and HR animals were faster to explore novel sections. This is in line with other studies reporting HR pigs to be more 'bold', i.e. less reluctant and faster than LR pigs to investigate novel stimuli (Hessing et al. 1994; Ruis et al. 2000; Bolhuis et al. 2004). LR pigs spent more time nosing than HR pigs during exploration trials, in line with previous proposals that LR pigs would be more 'thorough' explorers than HR pigs (Hessing et al. 1994; Ruis et al. 2001). In addition, our study is the first to suggest that LR and HR pigs also may differ in the way their behaviour changes after repeated exposures. Over trials, HR pigs showed a decrease in locomotion and alertness, whereas LR pigs were more constant in their locomotor behaviour over the three trials and even showed an increase in standing alert. This divergence in 'explorative strategy' has been identified as a major distinguishing factor between individuals differing in behavioural style in many species (Verbeek et al. 1994; Cools and Gingras 1998; Wilson 1998; Benus 2001).

HR pigs vocalised more frequently than LR pigs during exploration trials which is in line with their vocal response in other challenging situations (Ruis et al. 2001; Geverink et al. 2002). During observations, we noticed that the LR and HR pen mates maintained proximity or appeared to keep track of each other's location by vocalising. It is striking that HR and LR pigs differed in behaviour patterns and vocalisation frequency in spite of the apparent influence of their pen mates. It is likely that exposure to the maze in LR-LR or HR-HR pairs would have led to an even more marked effect of Backtest classification on behavioural and vocal responses.

\section{Memory tests}

During the first individual exposure to the maze (the habituation trial in which the shortest route to the exit was still available) all animals reached the exit within $30 \mathrm{~s}$, indicating that social reinstatement provides a high motivation to return to the home pen. It also indicates that pigs are able to solve this task rather easily, even though no apparent goal was present during exploration trials: reaching the waiting area during the exploration sessions did not directly allow the animals to return to their home pen and, furthermore, the exploration in pairs was aimed at reducing the motivation to return.
Due to the modification introduced into the familiar maze during MT1, pigs had to move away from the exit in order to reach it. Pigs varied largely in their ability to adapt their locomotor pattern: some animals made only a few incorrect line crossings and reached the exit within $2 \mathrm{~min}$, whereas seven pigs had to be guided to the exit after 15 min. However, despite large effects on exploration patterns, housing or Backtest classification did not affect performance in the memory tests much. HR animals did spend a higher percentage of time in persistent sections, i.e. sections that led to the exit during previous trials, in MT1 as compared to LR pigs, suggesting a higher tendency to form behavioural routines that rely on procedural memory processes, in line with previous findings (Bolhuis et al. 2004, 2005a). This difference did, however, not lead to a higher number of errors or a longer latency to reach the exit in HR animals.

Schmajuk et al. (1993) suggested hippocampal involvement in latent learning and detour spatial tasks, and the beneficial effects of environmental enrichment on hippocampal development have been well-described in rodents (Kempermann et al. 1997; Ickes et al. 2000), arguing against the suggestion that environmental enrichment does not affect latent learning. It cannot be ruled out that the enrichment provided in our study, i.e. the presence of rooting material, although profoundly affecting behaviour, was not sufficient to improve spatial cognitive performance in pigs. It should be noted, however, that changes in functional activity of the hippocampus in pigs from enriched conditions comparable to those in our study have been described (Van der Beek et al. 2000; Van der Beek et al., unpublished data).

The substantial improvement of performance in MT2 as compared to MT1 indicates that once a goal is apparent, pigs are able to solve a complex spatial memory task easily. In a previous study (Bolhuis et al. 2004), we found that although pigs differed in distractibility and behavioural flexibility in a T-maze, initial performance was not affected by behavioural style or rearing environment. These results, taken together with the results of previous spatial maze tasks (Sneddon et al. 2000; De Jong et al. 2000), indicate that in order to test spatial cognition in pigs, a more complex task is necessary. We therefore feel that latent learning, as tested in our experiment, may be a valuable method to assess spatial memory skills in pigs. As opposed to the radial arm maze experiments described by Mendl and colleagues in which working memory and procedural memory can be assessed in pigs (Laughlin and Mendl 2000; Laughlin et al. 1999), the utilization of a complex maze allows for testing hippocampal function. A recently described adapted Morris Water Maze for pigs (Siegford et al. 2008) offers similar possibilities, but is limited to use in very young animals, warranting further exploration of the novel methodology of the current study. 
In conclusion, although the performance of pigs in a novel latent detour task showed sufficient variability, and social reinstatement as an incentive to complete the task proved successful, neither housing conditions nor individual behavioural characteristics as measured by a Backtest affected spatial memory. However, the substantial difference in performance between memory test 1 and memory test 2 indicates that once a goal is apparent, pigs are able to complete a spatial task easily. Further exploration of our latent learning paradigm may therefore be of additional value for studying spatial memory. In addition, we found that both environmental enrichment and individual behavioural styles strongly affected exploration patterns, and that these differences were still present during memory testing. We recommend that future studies on spatial memory in pigs take this into account, as exploration strategies may be confused with memory performance.

Acknowledgments The Dutch law on animal experiments was followed, which complies with the ETS123 (council of Europe 1985) and the 86/609/EEC directive. The Institutional Animal Care and Use Committee approved the experiment. The authors would like to thank the personnel of experimental farm 'De Haar', Egbert Urff, Patrick H. Zimmerman, and T. Bas Rodenburg for technical assistance. This study was supported by the Earth and Life Sciences (ALW) council of the Netherlands Organisation for Scientific Research (NWO; grant ALW, PPWZ805-46.012).

Open Access This article is distributed under the terms of the Creative Commons Attribution Noncommercial License which permits any noncommercial use, distribution, and reproduction in any medium, provided the original author(s) and source are credited.

\section{References}

Beattie VE, O'Connell NE, Kilpatrick DJ, Moss BW (2000) Influence of environmental enrichment on welfare-related behavioural and physiological parameters in growing pigs. Anim Sci 70:443-450

Benus RF (2001) Coping in female mice from lines bidirectionally selected for male aggression. Behav 138:997-1008. doi:10.1163/ 156853901753286533

Benus RF, Bohus B, Koolhaas JM, Van Oortmerssen GA (1991) Behavioural differences between artificially selected aggressive and non-aggressive mice: response to apomorphine. Behav Brain Res 43:203-208. doi:10.1016/S0166-4328(05)80072-5

Bindu B, Alladi PA, Mansooralikhan BM, Srikumar BN, Raju TR, Kutty BM (2007) Short-term exposure to an enriched environment enhances dendritic branching but not brain-derived neurotrophic factor expression in the hippocampus of rats with ventral subicular lesions. Neuroscience 144:412-423. doi:10.1016/j.neuroscience.2006.09.057

Bolhuis JE (2004) Personalities in pigs : individual characteristics and coping with environmental challenges. Ph.D. thesis, Wageningen University, The Netherlands, pp 176

Bolhuis JE, Parmentier HK, Schouten WGP, Schrama JW, Wiegant VM (2003) Effects of housing and individual coping characteristics on immune responses of pigs. Physiol Behav 79:289-296. doi:10.1016/S0031-9384(03)00090-8

Bolhuis JE, Schouten WGP, De Leeuw JA, Schrama JW, Wiegant VM (2004) Individual coping characteristics, rearing conditions, and behavioural flexibility in pigs. Behav Brain Res 152:351-360. doi:10.1016/j.bbr.2003.10.024

Bolhuis JE, Schouten WGP, Schrama JW, Wiegant VM (2005a) Individual coping characteristics, aggressiveness and fighting strategies in pigs. Anim Behav 69:1085-1091. doi:10.1016/j.anbehav. 2004.09.013

Bolhuis JE, Schouten WGP, Schrama JW, Wiegant VM (2005b) Behavioural development of pigs with different coping characteristics in barren and substrate-enriched housing conditions. Appl Anim Behav Sci 93:213-228. doi:10.1016/j.applanim.2005.01.006

Bolhuis JE, Schouten WGP, Schrama JW, Wiegant VM (2006) Effects of rearing and housing environment on pigs with different coping characteristics. Appl Anim Behav Sci 101:68-85. doi:10.1016/ j.applanim.2006.01.001

Chapuis N (1987) Detour and shortcut abilities in several species of mammals. In: Ellen P, Thinus-Blanc C (eds) Cognitive processes and spatial orientation in animal and man. Nijhoff, Dordrecht, pp 97-106

Cools AR, Gingras MA (1998) Nijmegen high and low responders to novelty: a new tool in the search after the neurobiology of drug use liability. Pharmacol Biochem Behav 60:151-159. doi:10.1016/S0091-3057(97)00586-8

De Jong IC, Ekkel ED, Van de Burgwal JA, Lambooij E, Korte SM, Ruis MAW, Koolhaas JM, Blokhuis HJ (1998) Effects of strawbedding on physiological responses to stressors and behavior in growing pigs. Physiol Behav 64:303-310. doi:10.1016/S00319384(98)00066-3

De Jong IC, Prelle IT, Van de Burgwal JA, Lambooij E, Korte SM, Blokhuis HJ, Koolhaas JM (2000) Effects of environmental enrichment on behavioral responses to novelty, learning, and memory, and the circadian rhythm to cortisol in growing pigs. Physiol Behav 68:571-578. doi:10.1016/S0031-9384(99)00212-7

Freire R, Cheng HW, Nicol CJ (2004) Development of spatial memory in occlusion-experienced domestic chicks. Anim Behav 67:141150. doi:10.1016/j.anbehav.2003.03.015

Frick KM, Fernandez SM (2003) Enrichment enhances spatial memory and increases synaptophysin levels in aged female mice. Neurobiol 24:615-626. doi:10.1016/S0197-4580(02)00138-0

Geverink NA, Schouten WGP, Gort G, Wiegant VM (2002) Individual differences in behavioral and physiological responses to restraint stress in pigs. Physiol Behav 77:451-457. doi:10.1016/S00319384(02)00877-6

Geverink NA, Schouten WGP, Gort G, Wiegant VM (2003) Individual differences in behaviour, physiology and pathology in breeding gilts housed in groups or stalls. Appl Anim Behav Sci 81:29-41. doi:10.1016/S0168-1591(02)00253-8

Geverink NA, Heetkamp MJW, Schouten WGP, Wiegant VM, Schrama JW (2004) Backtest type and housing condition of pigs influence energy metabolism. J Anim Sci 82:1227-1233

Groothuis TG, Carere C (2005) Avian personalities: characterization and epigenesis. Neurosci Biobehav Rev 29:137-150. doi:10.1016/ j.neubiorev.2004.06.010

Gundlach H (1986) Brutfürsorge, Brutpflege, Verhaltensontogenese und Tagesperiodik beim Europäischen Wildschwein (Sus scrofa L.). Z Tierpsychol 25:955-995

Hammell DL, Kratzer DD, Bramble WJ (1975) Avoidance and maze learning in pigs. J Anim Sci 40:573-579

Haskell M, Wemelsfelder F, Mendl MT, Calvert S, Lawrence AB (1996) The effect of substrate-enriched and substrate-impoverished environments on the diversity of behaviour in pigs. Behav 133:741-761

Held S, Baumgartner J, Kilbride A, Byrne RW, Mendl M (2005) Foraging behaviour in domestic pigs (Sus scrofa): remembering and prioritizing food sites of different value. Anim Cogn 8:114-121. doi:10.1007/s10071-004-0242-y

Hessing MJC, Hagelsø AM, Van Beek JAM, Wiepkema PR, Schouten WGP, Krukow R (1993) Individual behavioural characteristics in 
pigs. Appl Anim Behav Sci 37:285-295. doi:10.1016/0168-1591 (93)90118-9

Hessing MJC, Hagels $\varnothing$ AM, Schouten WGP, Wiepkema PR, Van Beek JAM (1994) Individual behavioural and physiological strategies in pigs. Physiol Behav 55:39-46. doi:10.1016/0031-9384(94) 90007-8

Ickes BR, Pham TM, Sanders LA, Albeck DS, Mohammed AH, Granholm AC (2000) Long-term environmental enrichment leads to regional increases in neurotrophin levels in rat brain. Exp Neurol 164:45-52. doi:10.1006/exnr.2000.7415

Jensen P (1986) Observations on the maternal behaviour of freeranging domestic pigs. Appl Anim Behav Sci 16:131-142. doi:10.1016/0168-1591(86)90105-X

Kempermann G, Kuhn HG, Gage FH (1997) More hippocampal neurons in adult mice living in an enriched environment. Nature 386:493-495. doi:10.1038/386493a0

Koolhaas JM, De Boer SF, Buwalda B, Van der Vegt BJ, Carere C, Groothuis AGG (2001) How and why coping systems vary among individuals. In: Broom DM (ed) Coping with challenge. Welfare in animals including humans. Dahlem Workshop Report 87, Dahlem University Press, Berlin, pp 197-210

Laughlin K, Mendl M (2000) Pigs shift too: foraging strategies and spatial memory in the domestic pig. Anim Behav 60:403-410. doi: $10.1006 /$ anbe. 2000.1468

Laughlin K, Huck M, Mendl M (1999) Disturbance effects of environmental stimuli on pig spatial memory. Appl Anim Behav Sci 64:169-180. doi:10.1016/S0168-1591(99)00036-2

Leggio MG, Mandolesi L, Federico F, Spirito F, Ricci B, Gelfo F, Petrosini L (2005) Environmental enrichment promotes improved spatial abilities and enhanced dendritic growth in the rat. Behav Brain Res 163:78-90. doi:10.1016/j.bbr.2005.04.009

Lewis KP (2000) A comparative study of primate play behaviour: Implications for the study of cognition. Folia Primatol 71:417421. doi: $10.1159 / 000052740$

Mendl M, Erhard HW, Haskell M, Wemelsfelder F, Lawrence AB (1997a) Experience in substrate-enriched and substrate-impoverished environments affects behaviour of pigs in a T-maze task. Behav 134:643-659

Mendl M, Laughlin K, Hitchcock D (1997b) Pigs in space: spatial memory and its susceptibility to interference. Anim Behav 54:1491-1508. doi:10.1006/anbe.1997.0564

Meshi D, Drew MR, Saxe M, Ansorge MS, David D, Santarelli L, Malapani C, Moore H, Hen R (2006) Hippocampal neurogenesis is not required for behavioral effects of environmental enrichment. Nat Neurosci 9:729-731. doi:10.1038/nn1696

Morgan MJ (1973) Effects of post-weaning environment on learning in the rat. Anim Behav 21:429-442. doi:10.1016/S0003-3472(73) 80002-8

Newberry RC, Wood-Gush DGM (1988) Development of some behaviour patterns in piglets under semi-natural conditions. Anim Prod 46:103-109

O'Keefe J, Nadel L (1978) The Hippocampus as a Cognitive Map. Oxford University Press, New York

Olson AK, Eadie BD, Ernst C, Christie BR (2006) Environmental enrichment and voluntary exercise massively increase neurogenesis in the adult hippocampus via dissociable pathways. Hippocampus 16:250-260. doi:10.1002/hipo.20157

Olsson IAS, De Jonge FH, Schuurman T, Helmond FA (1999) Poor rearing conditions and social stress in pigs: repeated social challenge and the effect on behavioural and physiological responses to stressors. Behav Proc 46:201-215. doi:10.1016/S0376-6357(99) 00036-4

Petersen V (1994) The development of feeding and investigatory behaviour in free-ranging domestic pigs during their first 18 weeks of life. Appl Anim Behav Sci 42:87-98. doi:10.1016/ 0168-1591(94)90149-X
Poucet B, Thinus-Blanc C, Chapuis N (1983) Route planning in cats, in relation to the visibility of the goal. Anim Behav 31:594-599. doi:10.1016/S0003-3472(83)80083-9

Regolin L, Vallortigara G, Zanforlin M (1994) Perceptual and motivational aspects of detour behaviour in young chicks. Anim Behav 47:123-131. doi:10.1006/anbe.1994.1014

Regolin L, Vallortigara G, Zanforlin M (1995) Object and spatial representations in detour problems by chicks. Anim Behav 49:195199. doi:10.1016/0003-3472(95)80167-7

Renner MJ, Rozenzweig MR (1987) Enriched and impoverished environments. Effects on brain and behavior. Spinger, New York

Rosenzweig MR, Bennett EL (1996) Psychobiology of plasticity: effects of training and experience on brain and behavior. Behav Brain Res 78:57-65. doi:10.1016/0166-4328(95)00216-2

Ruis MAW, Te Brake JHA, Engel B, Buist WG, Blokhuis HJ, Koolhaas JM (2001) Adaptation to social isolation-acute and longterm stress responses of growing gilts with different coping characteristics. Physiol Behav 73:541-551. doi:10.1016/S0031-9384 (01)00548-0

Ruis MAW, Te Brake JHA, Van de Burgwal JA, De Jong IC, Blokhuis HJ, Koolhaas JM (2000) Personalities in female domesticated pigs: behavioral and physiological indications. Appl Anim Behav Sci 66:31-47. doi:10.1016/S0168-1591(99)00070-2

Schley L, Roper TJ (2003) Diet of wild boar Sus scrofa in Western Europe, with particular reference to consumption of agricultural crops. Mammal Rev 33:43-56. doi:10.1046/j.1365-2907.2003. 00010.x

Schmajuk NA, Thieme AD, Blair HT (1993) Maps, routes, and the hippocampus: a neural network approach. Hippocampus 3:387-400. doi:10.1002/hipo.450030312

Schouten WGP (1986) Rearing conditions and behaviour in pigs. $\mathrm{Ph} . \mathrm{D}$. Thesis. Agricultural University, Wageningen, pp 151

Schrijver NC, Bahr HI, Weiss IC, Würbel H (2002) Dissociable effects of isolation rearing and environmental enrichment on exploration, spatial learning and HPA activity in adult rats. Pharmacol Biochem Behav 73:209-224. doi:10.1016/S0091-3057(02)00790-6

Sneddon IA, Beattie VE, Dunne L, Neil W (2000) The effect of environmental enrichment on learning in pigs. Anim Welf 9:373-383

Siegford JM, Rucker G, Zanella AJ (2008) Effects of pre-weaning exposure to a maze on stress responses in pigs at weaning and on subsequent performance in spatial and fear-related tests. Appl Anim Behav Sci 110:189-202. doi:10.1016/j.applanim.2007. 03.022

Spinka M, Newberry RC, Bekoff M (2001) Mammalian play: training for the unexpected. Q Rev Biol 76:141-168

Tolman EC (1948) Cognitive maps in rats and men. Psychol Rev 55:189-208

Vallortigara G, Cailotto M, Zanforlin M (1990) Sex differences in social reinstatement motivation of the domestic chick (Gallus gal$l u s$ ) revealed by runway tests with social and nonsocial reinforcement. J Comp Psychol 104:361-367

Van der Beek EM, Bolhuis JE, Karman AG, Van Eerdenburg FJCM, Wiegant VM (2000) Housing condition affects the correlation between volume of the dentate gyrus and the cortisol response to stress in pigs. Abstracts Society for Neuroscience, vol 26, part 1. 30th Annual Meeting, New Orleans (abstract)

Verbeek MEM, Drent PJ, Wiepkema PR (1994) Consistent individual differences in early exploratory behaviour and dominance in juvenile male great tits. Anim Behav 48:1113-1121. doi:10.1006/ anbe.1994.1344

Wemelsfelder F, Haskell M, Mendl MT, Calvert S, Lawrence AB (2000) Diversity of behaviour during novel object tests is reduced in pigs housed in substrate-impoverished conditions. Anim Behav 60:385-394. doi:10.1006/anbe.2000.1466

Wickert DA, Barr GR (1966) Studies of learning ability in young pigs. J Anim Sci 25:1280 
Williams BM, Luo Y, Ward C, Redd K, Gibson R, Kuczaj SA, McCoy JG (2001) Environmental enrichment: effects on spatial memory and hippocampal CREB immunoreactivity. Physiol Behav 73:649-658. doi:10.1016/S0031-9384(01)00543-1

Wilson DS (1998) Adaptive individual differences within single populations. Philos Trans R Soc Lond B 353:199-205

Wynne CDL, Leguet B (2004) Detour behavior in the Quokka (Setonix brachyurus). Behav Proc 67:281-286. doi:10.1016/j.beproc. 2004.04.007

Young D, Lawlor PA, Leone P, Dragunow M, During MJ (1999) Environmental enrichment inhibits spontaneous apoptosis, prevents seizures and is neuroprotective. Nat Med 5:448-453. doi: $10.1038 / 7449$

Zimmermann A, Stauffacher M, Langhans W, Wurbel H (2001) Enrichment-dependent differences in novelty exploration in rats can be explained by habituation. Behav Brain Res 121:11-20. doi:10.1016/S0166-4328(00)00377-6

Zucca P, Antonelli F, Vallortigara G (2005) Detour behaviour in three species of birds: quails (Coturnix sp.), herring gulls (Larus cachinnans) and canaries (Serinus canaria). Anim Cogn 8:122-128. doi:10.1007/s10071-004-0243-x 